\title{
Will In Vitro Tests Replace Animal Models in Experimental Oncology?
}

\author{
Martyna Wesserling ${ }^{1}$, Tomasz Drewa ${ }^{1,2 *}$
}

${ }^{1}$ Department of Tissue Engineering, University Bydgoszcz, Poland

${ }^{2}$ Department of Urology, Institute of Oncology, Bydgoszcz, Poland

\begin{abstract}
Every year many people die of cancer. Hopes give the extensive studies conduct worldwide on the new anticancer agents. New drugs are tested in both, in vitro and in vivo conditions. This model is recognized as a standard all around the world. On the other hand many organizations attempt to decrease experimental work on animal models, suggesting that in vivo tests are unethical and probably useless. We would like to show the differences between in vitro and in vivo conditions. This review is aimed to emphasized the differences related to receptors, hormones, bioavailability, enzymatic influence, vasculature, age, severity of disease and contamination of cell lines. It seems that it is not possible to abolish in vivo studies in testing any anticancer agent nowadays. The question is how extensive experiments on animal should be?
\end{abstract}

\section{Introduction}

The action of new drugs is not always fully understood, even if the drug is regular prescribed and used in clinical condition. The history of Thalidomide or Rofecoxib had implicated that we should understand and take into account the difficulties in predicting many side effects.

Cell cultures in vitro attempt to explain ambiguities that appear during the experimental work. Studies on cells isolated from the living organism and established cancer cell lines are used to assess the safety and possible mechanisms of future anticancer substances. However, various models for such research need to be used. The examples being: experimental model in vitro, animal studies, studies on humans, and clinical safety and effectiveness. The fact that we do not always understand the mechanism of tested substances or new drugs in vivo appears to be the most significant obstacle in tests on humans and clinical assessment. Nevertheless the use of drug called thalidomide between 1950 and 1960 demonstrated how unpredictable effects may result from the existence of differences between in vitro and in vivo studies. Thalidomide was used to ameliorate nausea and vomit during pregnancy. Drug was withdrawn from the market due to limb malformations and abnormalities of internal organs in the newborns. However, the clinical research on the re-use of this drug in treatment of patients suffering from renal cell carcinoma and recurrent high-grade gliomas is now in progress [1-3]. Thalidomide inhibits angiogenesis on an animal. This mechanism can not be observed in vitro [4]. Unexpected effects occurred while using rofecoxib were also observed. Rofecoxib was used as a painkiller in treatment of patients suffering from osteoarthritis. In 2004 drug was withdrawn from the market because it contributed to the death of patients suffering from ischemic cardiac disease $[5,6]$. This fatal complication can not be tested in vitro and probably even animal model will not help to predict this side effect. Even, if we noticed the clinical effectiveness of the drugs, the real effects would be different and startling.

These examples show that it is essential and important to emphasized the differences between in vivo and in vitro studies. It is very important to show and analyze the drawbacks of in vitro testing. In this study we review the differences between in vitro and in vivo models related to receptors, hormones, bioavailability, enzymatic influence, blood supply, age of tested cells, severity of disease and contamination of cell lines. Based on this analysis we try to answer on question from title - Will in vivo models be ever replaced by in vitro testing?

\section{Receptor status and in vivo models}

The existence of specific receptors detected in vivo determines visible differences in experiments on possible anticancer drugs. Yu et al noticed that restoring androgen receptor (AR) in PC3 cells resulted in decreased proliferation and invasion/metastasis ability tested in MTT, soft agar, chamber invasion and wound healing assay [7]. PC3, isolated from prostate cancer bone metastasis, is an androgen independent human prostate cancer cell line. In the mouse orthotopic xenograft model, PC3-AR9 (a PC3 stable clone expressing human AR driven by natural human AR promoter) resulted in smaller primary tumors and metastasis tumors, with a lower proliferation rate and higher apoptosis rate. This cancer cell line (PC3) can answer for the treatment differentially depending on receptor status. It is known that prostate cancer is highly heterogenous tumor, showing different expression of $\mathrm{AR}$ in one patient. This fact is usually neglected in many in vitro studies [7].

Repifermin (140 amino acids polypeptide) is a truncated form of recombinant Keratinocyte Growth Factor-2 (KGF-2) that retains the pharmacological and biological activity of natural KGF-2 [8]. Repifermin selectively induces epithelial cell proliferation, differentiation and migration. Repifermin stimulated neither in vitro nor in vivo growth of tumors of the pharynx (cell lines Detroit 562 and $\mathrm{FaDu}$ ) and cancer of the tongue (cell line SCC-25). In this regard, it's influence is different from epithelial growth factor (EGF), which has been shown to stimulate growth of selected tumors [9]. That means that receptor status can explain differences between in vivo and in vitro studies. The status of receptors in cell culture can be changed and modulated in vivo. Paracrine influence form other cell population can stimulate cells to grow in vivo but this effect can be abolish in monoculture. Cells in culture lose characteristic interactions appropriate for tissue and live in different

*Corresponding author: Tomasz Drewa, MD, PhD, FEBU, Department of Tissue Engineering Nicolaus Copernicus University, ul. Karlowicza 24, 85-092 Bydgoszcz, Poland, Tel: 0048525853737; Fax: 0048525853742; Email: tomaszdrewa@wp.pl

Received April 09, 2011; Accepted April 09, 2011; Published May 06, 2011

Citation: Wesserling M, Drewa T (2010) Will In Vitro Tests Replace Anima Models in Experimental Oncology? J Tissue Sci Eng 2:102e. doi:10.4172/21577552.1000102e

Copyright: (C) 2010 Wesserling M, et al. This is an open-access article distributed under the terms of the Creative Commons Attribution License, which permits unrestricted use, distribution, and reproduction in any medium, provided the original author and source are credited. 
environment. This may affect the different status of the receptor in vitro and in vivo. In a controlled in vitro assay, we can change the expression of only selected receptors. These effects can not be achieved in vivo. We need to know whether the terms of the experiment have an impact on or any relationship with expression of receptors. These differences may have a significant influence on cells sensitivity for tested drug, cell proliferation and ability to induce apoptosis within them.

\section{Sex hormones and in vitro cell growth}

Hormonal influences may lead to carcinogenic risk. The growth and proliferation of breast and prostate cancers can be driven by their respective classes of steroid hormones estrogens and androgens by binding to their cognate receptors, but no correlation between hormone level and hormone-related cancer incidence exists. One the other hand one of the great therapeutic successes in cancer treatment was the development of tamoxifen, an estrogen receptor (ER) antagonist that is well tolerated and effective in patients with ER-positive breast cancer [10]. It has to be emphasized that hormonal influence can be fully observed in vivo, due to the fact that tumor has heterogenous population of cells, which reflects process of cells maturating. On the other hand established cancer cell lines show homogenous morphology and probably function. The current concept of in vitro experiments require on homogenous population of cells regarding their maturation.

Differences between sexes is the reason that in animal carcinogenesis experiments both sexes of each species are usually tested. Germann et al observed that the mortality reflected a typical dose-response effect. Isophosphoramide mustard (the cytotoxic alkylating metabolite of Ifosfamide) is slightly more toxic in females than in males [11]. Chipman et al comprised lung tumors from male and female patients and identified various tumor characteristics that may affect tumor etiology in a gender-specific manner. It has to be emphasized that lung cancer is not hormone related one [12]. It is impossible to simulate in vitro an environment of cancer bearing organism, so the in vitro experiment will not give answer on these important questions related to gender influence. Usually one cell line is used in experiment and additionally nobody cares about cell line origin with respect to sex and hormonal status of the host organism. Particularly, carcinogenesis within the lungs may be modulated depending on exposure to estrogen [12]. Estrogen-induced cell proliferation is a critical step in the etiology and progression of a variety of tumor types. The cellular response to estrogen is mediated by estrogen receptor $\alpha$ and $\beta$ (ER- $\alpha$ and ER- $\beta$ ). These receptors function as ligand-dependent transcription factors and regulate the expression of genes implicated in cell cycle control, signal transduction, and cell survival [12]. This difference develops as animals reach sexual maturity and seems to be mediated mainly by estrogens. Allen et al noticed strong associations between concentrations of sex hormones and endometrial cancer risk [13]. These analyses show that estrogens and testosterone are probably positively associated with an increased risk of endometrial cancer in postmenopausal women [13]. It was shown that hormonal factors are involved in the etiology of cancers. The hormonal status of the host organism has a critical influence on the in vivo experiment. This fact has an great impact on weakness of in vitro experimental work.

\section{Bioavailability and detoxification cannot be tested in vitro}

Rate of absorption of any anticancer substances depends on different routes of administration. Drug given orally enters to bloodstream and reached target tissues slower than intravenously. During intravenous dosing time of drug absorption practically has no meaning. In track of single circulation of medicine within blood a big part of introduced substance becomes disposed in other tissues, and the dose reaching the tumor is usually relatively small. Direct intratumor drug injection (via a nutrition vessel) can help to overcome this negative effect. Location of tumor and mode of delivery has also big value in search of new anticancer substances. Eilon et al in experiment about the potential efficacy of epoxide-containing piperazines as a new class of anti-cancer agents, has been observed the differing bioavailability and anti-cancer activity of the two drugs when tested xenograft tumors in mice or human tumors grown under the kidney capsule in mice [14] Breast and prostate cancer cells were grown in the renal capsule. It is place where organism immunological response can not be found and the influence of natural chemokines and hormones were limited. It has been also noticed lower bioavailability of hydrophobic compound when administered in water [14]. It can be concluded that water environment has a great impact on drug bioavailability. This environment can be slightly different in many tissues.

Enhanced Protein Tyrosine Kinase (PTK) activity correlates with the development of cancer and other proliferative diseases. The hypothesis that PTK inhibitors may be of value in the treatment of cancer led to the systematic synthesis of selective tyrosine phosphorylation inhibitors (tyrphostins) that show in vitro and in vivo anticancer activity [15]. Li et al noticed that it was difficult to extrapolate in vitro results to predict in vivo situations, species differences in biostability of the potential anticancer agent, adaphostin (a tyrphostin analog that inhibits bcr/abl) may be related to its therapeutic and toxic effects. Since adaphostin is most stable in human and mouse plasma, the mouse model may be better used to predict pharmacologic and toxicologic profiles then other animal models. An intraperitoneal dose was greater than that following a subcutaneous dose, or that for a dose administered orally. The lower bioavailability following oral and subcutaneous dosing may limit the use of these important routes of administration. The extremely low bioavailability following oral administration is probably due to minimal absorption of precipitated drug [16]. Song et al showed that after intraperitoneally administration only $1.5 \%$ of the roscovitine (a purine analogue and cyclin-dependent kinase inhibitor) was detected in the bone marrow. Thus, the roscovitine effect on hematopoietic progenitors in bone marrow in vivo can be only transient. One reason may be that only a small fraction of this drug reaches the bone marrow [17]. Lin et al in their experimental studies on tissue distribution of arsenic showed that parenteral administration of arsenic trioxide has recently been recognized as an effective antineoplastic therapy, especially for the treatment of acute promyelocytic leukemia [18] The main results of this study using a rabbit model were as As(III) accumulated within tissue selectivity after multiple chronic parenteral arsenic trioxide administration and could be washout completely in bladder, partially in liver, heart, lung, and kidney or minimally in hair This study demonstrated nonlinear blood levels of As (III) following parenteral administration of $\mathrm{As}_{2} \mathrm{O}_{3}$. It has been demonstrated important role of enzymes in vivo. Efficient metabolizing enzyme systems convert As (III) to DMA. Thus, DMA was the major metabolite in tissue after $\mathrm{As}_{2} \mathrm{O}_{3}$ therapy. Nonetheless, the over-saturation sustained at high doses of $\mathrm{As}_{2} \mathrm{O}_{3}$ may be compensated by enzyme induction in certain tissues (e.g., kidney). The tissue accumulation of arsenic compounds and its reversibility after washout were tissue-selective. The potential for late toxicities of $\mathrm{As}_{2} \mathrm{O}_{3}$ in organs with a significant tendency for arsenic accumulation and low reversibility [18]. Tabaru et al found a high concentration of oily anticancer agents in the pleural or peritoneal cavities for a few weeks and a low drug concentration in the blood stream after intracavitary administration. Oily anticancer agents showed definite antitumor activity against peritoneal cancer dissemination. 
However intraperitoneal injection of oil anticancer agents alone in rabbits with enlarged tumor did not prevent peritoneal dissemination. Although the same drug and the same dosage were used in two groups of rabbits, their survival periods were significantly different [19]. These results showed that in vivo studies are very important in searching new anticancer drugs. Complicated system like a living organism, its metabolism and the influence of many enzymes, affect the different of drug bioavailability and distribution. It is impossible to avoid in vivo studies in testing any anticancer drug till now.

Ikeda showed on the experimental renal failure, plasma clearance of 5-chloro-2,4- dihydroxypyridine (CDHP) and 5-fluorouracil (5-FU) was retarded corresponding to the degree of renal impairment and was close correlated with creatinine clearance and plasma CDHP and 5-FU clearance. In contrast, in patients with mild and moderate renal dysfunction the T1/2 values of CDHP with consecutive administrations were longer than the values with single administration. In the clinical setting, the T1/2 and the AUC of 5-FU in patients with renal impairment were longer and greater than in patients with normal renal function [20]. It is important to noticed that experiment in vitro does not provide for the origin of the cell line. In in vitro studies nobody does not pay attention, whether the tumor cells come from. Whether the cell line was derived healthy or sick organism. These factors may be significant in predicting on organism reaction for drug.

\section{Vasculature}

Tumor vasculature is a important factor leading to drug resistance. It has been noticed that angiogenesis is a key factor involved in the development and progression of human tumors [21,22]. Vascular endothelial cell growth factor (VEGF) enhances growth of new vessels (angiogenesis) [22]. Angiogenesis appears to be essential for the growth of solid tumors and their metastasis. VEGF plays a principal role of angiogenesis within tumor (neo-angiogenesis) [22-24]. Angiogenesis is a vital process resulting in the formation of new blood vessels from existing vasculature [24]. Plays an essential role in physiological and pathological phenomena, including embryonic development, wound healing, solid tumor growth, diabetic retinopathy, psoriasis, and rheumatoid arthritis and many other [22]. The VEGF growth factors and their receptors are the most important mediators of tumors angiogenesis [25]. Rahman et al distinguish two VEGF tyrosine kinases receptors: VEGFR-1 and VEGFR-2 [25]. Vascular endothelial growth factor (VEGF) is best known as a cytokine essential for embryonic vasculogenesis and for the angiogenesis associated with various pathologies including cancer [23]. This importance of VEGF suggests that blockade of its function may be useful for inhibiting angiogenesis and tumor growth [22]. Lin et al observed that levels of VEGF activity in the proximity between tumor cells and endothelial cells would be expected to inhibited by fascaplysin a marine natural product with CDK4 inhibitory activity in vivo. This action can not be fully tested in vitro, due to no sufficient model of 3-dimensional culture. The inhibitory effect on endothelial cells proliferation of fascaplysin is more significant in inhibition in vivo than in vitro due to a specific response of down-regulating VEGF secretion in vivo [21]. Good tumor vasculature warrants better answer for chemotherapy. It has been noticed vessel wall within the tumor tissue is more permeable to macromolecules than normal vasculature [12]. Low vascular density of the central part of the solid tumor leads to hypoxia in this region. The cells from central part are resistant to apoptosis due to high expression of antiapoptotic regulators [26]. Therapy of these tumors needs higher doses of anticancer drugs, but drug entering into this region is decreased due to weak blood supply. Cytotoxic effect obtain during an in vitro will never reflect the impact of tumor size, blood volume and supply.

\section{Age and severity of disease}

Some cancers are bound up with aging. These include breast, uterine, prostate, thyroid, pancreas, lung cancers. Uterine cancer occurs primarily in elderly women, the median age of onset being around 60 years old; only $5 \%$ of cases develop before age $40[10,27]$. That we noticed at the beginning, lung tumor genesis may be modulated depending on exposure to estrogen. Younger, presumably pre-menopausal, women with breast cancer appear to have shorter survival than older women [12]. Prostate cancer accounts for about 200,000 deaths annually worldwide, predominantly afflicting older men in developed countries. Prostate cancer tends to develop in men over the age of fifty while the peak of 70 years old $[10,27]$. More than any other, prostate cancer is one occurs in elderly. The incidence of pancreas cancer is so strongly agedependent [28]. Bladder cancer risk increases approximately linearly with duration of smoking, reaching a fivefold risk after 40 years, so the age relation is weaker $[10,27]$.

In addition, occur attendant diseases makes the divergence between the in vitro and in vivo studies. Lowenfels and coworkers noticed that two diseases increase the risk of pancreatic cancer: pancreatitis, and diabetes [29]. Also Zhou et al observed significant increases in deaths from cancer of the stomach, colon-rectum and liver in men with diabetes, and deaths from cancers of the liver and pancreas in women with diabetes [30]. In group of patients with alcoholic cirrhosis pancreatic cancer risk is significantly high [31]. Oral cavity cancers occur more often in patients with cirrhosis [31]. Along with life expectancy and duration, people are more expose to various risk factors or the simultaneous influence of several risk factors for cancer, which can strengthen existing genetic predisposition. Cell lines derived from young patients will be completely different than those derived from older person. Studies using cells derived from the 60 years person may have a real relationship with the effects on the treatment of prostate or pancreas cancer. It is important to pay attention that experiments on cells from young women would be totally different from studies on cells from post menopausal women. Among other things, the absence of such control does not allow to avoid in vivo studies. Age of tissue used for cell line establishment has great meaning if we want predict drug anticancer activity in vivo.

\section{Contaminations of cell lines}

Crosscontamination, in which the contaminant is another cell line, was first recognized in the 1950s but, unfortunately, is a serious problem today. Many experimental work has been performed on the contaminant cell lines. It has been resulted false conclusion. Crosscontamination may arise due to several causes, including spread via aerosols or accidental contact, use of unplugged pipets, sharing media and reagents among cell lines, and use of mitotically inactivated feeder layers or conditioned medium, which may carry contaminating cells if not properly eliminated e.g., by freeze-thaw and filtration [32-34]. Cross-contamination may occur "early", in which case the original cell line has probably never existed independently, or "late", where the tested sample has been overgrown but other stocks of the original may still exist [32]. Drexler and coworkers consider the two biggest problems in cell culture of continuous cell lines involve contaminations. The first is contamination with microorganisms, in particular with mycoplasmas, and the second is cross-contamination with other cell lines. It has been estimated that more than one-third of cell cultures in use are cross-contaminated either with cells from other species (interspecies contamination) or with unrelated cells from the same species (intraspecies contamination) [34]. Also very important 
factors responsible for the bias of in vitro studies are; contamination with viruses and genetic drift after manifold passage. Experiment has shown that high passage number has an adverse effect on murine bone marrow mesenchymal stem cells activation and protection [33]. Such cell cultures may be grown, maintained, and used for years, and results may be published without documented authenticity of the cells. The potential problems and even dangers in using cross-contaminated cell cultures for the quality of research and production in virtually any scientific or biomedical area cannot be overemphasized [34]. Cell line contamination is a serious issue that detracts from the use of cell lines as model systems to help us understand a broad range of diseases, including cancer. Responding practically by checking each cell line before it is used, searching for previous references and authenticating the sample itself, is worthwhile and will reduce the risk, and subsequent consequences, of contamination long-term [32]. Drexler et al. points out that the problem may be solved or at least mitigated by increasing the awareness of its seriousness and by introducing adequate identity control of cell lines. Vigilance in this area is clearly needed to avoid recurrence of past errors and the resulting confusion and waste of time/ money that they generate [34]. Cell lines are basic components of in vitro experiments and should be quality controlled and given the same care that buffers, enzymes, growth medium and other products used in laboratory.

\section{References}

1. Thomas DA, Kantarjian HM (2001) The revitalization of thalidomide. Ann Oncol 12: $885-886$

2. Minor D, Monroe D, Damico L, Meng G, Suryadevara U, et al. (2002) A phase II study of thalidomide in advanced metastatic renal cell carcinoma. Invest New Drugs 20: 389-393.

3. Fine H (1995) Novel biologic therapies for malignant gliomas. Antiangiogenesis, immunotherapy, and gene therapy. NeurClin 13: 827-846.

4. Moreira A, Friedlander D, Shif B, Kaplan G, Zagzag D (1999) Thalidomide and a thalidomide analogue inhibit endothelial cell proliferation in vitro. J Neurooncol 43: 109-114.

5. Beller G (2005) Lessons to be learned from the Vioxx debacle. J Nucl Cardiol 12: $1-2$.

6. Jørgensen A, Jørgensen K, Gøtzsche P (2010) Unbalanced reporting of benefits and harms in abstracts on rofecoxib. Eur J Clin Pharmacol 66: 341-347.

7. Yu SQ, Han BM, Shao Y, Wu JT, Zhao FJ, et al. (2009) Androgen receptor functioned as a suppressor in the prostate cancer cell line PC3 in vitro and in vivo. Chin Med J 122: 2779-2783.

8. Freytes CO, Ratanatharathorn V, Taylor C, Abboud C, Chesser N, et al. (2004) Phase $1 / I I$ randomized trial evaluating the safety and clinical effects of repifermin administered to reduce mucositis in patients undergoing autologous hematopoietic stem cell transplantation. Clin Cancer Res 10: 8318-8324.

9. Alderson R, Gohari-Fritsch S, Olsen H, Roschke V, Vance C, et al. (2002) In vitro and in vivo effects of repifermin (keratinocyte growth factor-2, KGF2) on human carcinoma cells. Cancer Chemother Pharmacol 50: 202-212

10. Boyle P, Levin B (2008) World Cancer Report 2008. France International Agency for Research on Cancer.

11. Germann N, Urien S, Rodgers A, Ratterree M, Struck R, et al. (2005) Comparative preclinical toxicology and pharmacology of isophosphoramide mustard, the active metabolite of ifosfamide. Cancer Chemother Pharmacol 55: 143-151.

12. Chipman S, Oldham F, Pezzoni G, Singer J (2006) Biological and clinical characterization of paclitaxel poliglumex (PPX, CT-2103), a macromolecular polymer-drug conjugate. Int J Nanomedicine 1: 375-383.

13. Allen NE, Key TJ, Dossus L, Rinaldi S, Cust A, et al. (2008) Endogenous sex hormones and endometrial cancer risk in women in the European Prospec- tive Investigation into Cancer and Nutrition (EPIC). Endocr Relat Cancer 15 485-97.

14. Eilon GF, Gu J, Slater LM, Hara K, Jacobs JW (2000) Tumor apoptosis induced by epoxide-containing piperazines, a new class of anti-cancer agents. Cancer Chemother Pharmacol 45: 183-191.

15. Levitzki A (2002) Tyrosine kinases as targets for cancer therapy. Eur J Cancer 38: 11-18

16. Li M, Wang H, Hill DL, Stinson S, Veley K, et al. (2006) Preclinical pharmacology of the novel antitumor agent adaphostin, a tyrphostin analog that inhibits bcr/abl. Cancer Chemother Pharmacol 57: 607-614.

17. Song H, Vita M, Sallam H, Tehranchi R, Nilsson C, et al. (2007) Effect of the Cdk-inhibitor roscovitine on mouse hematopoietic progenitors in vivo and in vitro. Cancer Chemother Pharmacol 60: 841-849.

18. Lin CJ, Wu MH, Hsueh YM, Sun SS, Cheng AL (2005) Tissue distribution of arsenic species in rabbits after single and multiple parenteral administration of arsenic trioxide: tissue accumulation and the reversibility after washout are tissue-selective. Cancer Chemother Pharmacol 55: 170-178.

19. Tabaru K, Konno T, Oda T, Nagamitsu A, Ishimaru Y, et al. (2001) Treatment of VX2 carcinoma implanted in the liver with arterial and intraperitoneal administration of oily anticancer agents. Cancer Chemother Pharmacol 47: 149-154.

20. Ikeda M, Furukawa H, Imamura H, Shimizu J, Ishida H, et al. (2002) Pharmacokinetic study of S-1, a novel oral fluorouracil antitumor agent in animal mode and in patients with impaired renal function. Cancer Chemother Pharmacol 50: 25-32.

21. Lin J, Yan XJ, Chen HM (2007) Fascaplysin, a selective CDK4 inhibitor, exhibit anti-angiogenic activity in vitro and in vivo. Cancer Chemother Pharmacol 59 439-445.

22. Ueda Y, Yamagishi T, Samata K, Ikeya H, Hirayama N, et al. (2004) A nove low molecular weight VEGF receptor-binding antagonist, VGA1102, inhibits the function of VEGF and in vivo tumor growth. Cancer Chemother Pharmacol 54 $16-24$

23. Singer D (2010) Vascular Endothelial Growth Factor: Much More than an Angiogenesis Factor. Mol Biol Cell 21: 377-379.

24. Nussenbaum F, Herman IM (2010) Tumor angiogenesis: insights and innovations. J Oncol.

25. Rahman R, Smith S, Rahman C, Grundy R (2010) Antiangiogenic therapy and mechanisms of tumor resistance in malignant glioma. $\mathrm{J}$ Oncol.

26. O’Reilly T, Wartmann M, Brueggen J, Allegrini PR, Floersheimer A, et al. (2008) Pharmacokinetic profile of the microtubule stabilizer patupilone in tumor-bearing rodents and comparison of anti-cancer activity with other MTS in vitro and in vivo. Cancer Chemother Pharmacol 62: 1045-1054.

27. Thomas A (2003) World Cancer Report 2003. France International Agency for Research on Cancer. IARCPress.

28. Lowenfels AB, Maisonneuve P (2006) Epidemiology and risk factors for pancreatic cancer. Best Pract Res Clin Gastroenterol 20: 197-209.

29. Lowenfels AB, Maisonneuve P (2005) Risk factors for pancreatic cancer. J Cell Biochem 95: 649-56

30. Zhou XH, Qiao Q, Zethelius B, Pyörälä K, Söderberg S, et al. (2010) Diabetes, prediabetes and cancer mortality. Diabetologia 53: 1867-76.

31. Goldacre MJ, Wotton CJ, Yeates D, Seagroatt V, Collier J (2008) Liver cirrhosis other liver diseases, pancreatitis and subsequent cancer: record linkage study. Eur J Gastroenterol Hepatol 20: 384-92.

32. ICapes-Davis A, Theodosopoulos G, Atkin I, Drexler HG, Kohara A, et al. (2010) Check your cultures! A list of cross-contaminated or misidentified cell lines. Int J Cancer 127: 1-8.

33. Crisostomo PR, Wang M, Wairiuko GM, Morrell ED, Terrell AM, et al. (2006) High passage number of stem cells adversely affects stem cell activation and myocardial protection. Shock 26: 575-80.

34. Drexler H, Dirks W, MacLeod R (1999) False human hematopoietic cell lines: cross-contaminations and misinterpretations. Leukemia 13: 1601-1607. 